\title{
Revisión sistemática de pruebas para obtener los parámetros geotécnicos del suelo: tendencias, alcances y limitaciones
}

Systematic review of test to obtain the geotechnical parameters of the
soil: ntrends, scopes and limitations

Recibido: noviembre 08 de 2021 | Revisado: noviembre 22 de 2021 | Aceptado: diciembre 02 de 2021

\author{
Alex D. Melchor Porras \\ Juan de Dios Malpartida ${ }^{\mathrm{I}}$ \\ Sócrates P. Muñoz PÉrez ${ }^{\mathrm{I}}$
}

\begin{abstract}
Resumen
El presente trabajo tiene como objetivo hacer una revisión de los ensayos que se realizan in situ para obtener los parámetros geotécnicos de un terreno. En primer lugar, se inicia con la descripción de los mismos exponiendo la problemática respecto a su aplicación en el tipo de suelo y luego se detalla cada uno de estos tales como SPT, CPT, DPSH, DPL y corte con veleta especificando desde el día que estos ensayos se iniciaron en la aplicación geotécnica como también su utilización. Estos ensayos aplicados in situ son muy importantes por lo que es necesario conocer cada uno de ellos desde su inicio y los cambios que han tenido en la aplicación geotécnica hasta la actualidad.
\end{abstract}

Palabras clave: ensayo DPL; ensayo DPSH; ensayo corte con veleta

\begin{abstract}
The objective of this work is to review the tests that are carried out in situ to obtain the geotechnical parameters of a terrain. In the first place, it begins with the description of the same, exposing the problem regarding its application in the type of soil and then each one of these such as SPT, CPT, DPSH, DPL and vane cutting test specifying from the day that these trials began in the geotechnical application as well as its use. These tests applied in situ are very important, so it is necessary to know each one of them from the beginning and the changes that they have had in the geotechnical application until today.
\end{abstract}

Key words: DPL test; DPSH test; vane cutting test
Autor para correspondencia

E-mail: msocrates@crece.uss.edu.pe

1 Universidad Señor de Sipán. Pimentel, Perú, Facultad de Ingeniería, msocrates yrbanismo.

msocrates@crece.uss.edu.pe

\author{
Cy words. DPL test; DPSH test; vane cutting test
}

\begin{abstract}
(C) Los autores. Este artículo es publicado por la Revista Campus de la Facultad de Ingeniería y Arquitectura de la Universidad de San Martín de Porres. Este artículo se distribuye en los términos de la Licencia Creative Commons Atribución No-comercial - Compartir-Igual 4.0 Internacional (https://creativecommons.org/licenses/ CC-BY), que permite el uso no comercial, distribución y reproducción en cualquier medio siempre que la obra original sea debidamente citada. Para uso comercial contactar a: revistacampus@usmp.pe.
\end{abstract}

https: 


\section{Introducción}

Existen diferentes maneras para obtener las muestras de un suelo mediante ensayos in situ. Se conoce una gran variedad que constituyen una serie de técnicas en su aplicación (DíazCuriel et al., 2017). En la actualidad, las construcciones de los diferentes tipos de obras civiles demandan realizar estudios geotécnicos cuyos parámetros se pueden obtener mediante ensayos y para lo cual existen varios métodos (Hipólito-Ojalvo et al., 2019).

Skempton (1986) especifica que entre los ensayos realizados en el sitio de estudio denominado in situ y los ensayos que se realizan en laboratorios existe concordancia de forma que en la prueba de laboratorio faciliten la interpretación de una forma completa a los ensayos que son realizados en el lugar de estudio in situ, y, por otra parte, las pruebas de equipos in situ se basan en evaluar la calidad de las muestras que han sido obtenidas por los diferentes métodos que existen.

En este contexto es importante mencionar que las condiciones geológicas de un lugar donde hay complejidad como por ejemplo las gravas en los suelos arcillosos, el ensayo de penetración SPT es inaceptable. De la misma manera, el ensayo de penetración de cono CPT que es empleado en suelos relativamente débiles que no contengan mezcla de grava y guijarros (Žaržojus et al., 2013) como es en el caso de Lituania que contiene un suelo con bastante gravas y guijarros, y en algunos casos estratos completos que no permiten la correcta aplicación de estos equipos.
La prueba de penetración estándar (SPT) es la prueba in situ comúnmente utilizada en Tanzania, pero se necesita mucha inversión de tiempo para aplicarla. Los ingenieros geotécnicos de Tanzania han hecho correlaciones de la prueba SPT con la prueba de penetración dinámica ligera (DPL) para aumentar la cantidad de información. Las investigaciones indicaron una correlación bastante buena a diferentes profundidades. En todo el mundo la prueba DPL es menos popular en comparación con la prueba SPT; sin embargo, la prueba DPL tiene varias ventajas sobre la prueba SPT como simplicidad y continuidad. Es una prueba rápida y de bajo costo de inversión. El principal inconveniente con la prueba DPL es el hecho de que en la mayoría de los casos no puede ser aplicable a profundidades superiores a $8 \mathrm{~m}$ (Lingwanda, Larsson y Nyaoro, 2015).

Reyes-Ortiz (2009) sostiene lo siguiente acerca del ensayo dinámico DPSH. Su ejecución es de forma continua, pero este tipo de ensayo tiene su límite de aplicación en el campo para exploración geológica ya que no puede ser aplicado en suelos que contengan rocas. De la misma manera, no es recomendable en suelos cementados entre gravas y arenas densas. (Khodaparast, Rajabi, \& Mohammadi, 2015) en la Región Central y Sur de Irán aplicaron el ensayo de DPSH para suelos de arcilla limo y arena para ver el comportamiento del sitio

Por otro lado, el ensayo de penetración dinámica ligero DPL es un ensayo que permite determinar la capacidad potente del suelo, pero como todos los equipos que son aplicados en campo in situ no es recomendable para todo tipo de suelo 
(Lingwanda et al., 2015). Como es en Tanzania hicieron una investigación geotécnica con el ensayo DPL para la estratificación del suelo.

Para medir la resistencia al corte no drenado en suelos cohesivos está el ensayo de corte con veleta. Este tipo de ensayo no puede ser aplicado en suelos que permiten el drenaje donde hay dilatación en el momento del ensayo y en suelos que contengan piedras ya que estas puede afectar en los resultados como en las arenas y limos (Cabalar, Mukhlif y Isik, 2020). En Turquía realizaron el ensayo de corte con veleta para determinar las características del sitio en suelos cohesivos no drenados.

Çoşkun, Sarışık, y Sarışık, (2017) especifican que la Mecánica de Suelos de la forma como se conoce, actualmente, se apoya sobre los ensayos de laboratorio como en los ensayos in situ.

El objetivo de este artículo es contextualizar una revisión sistemática de las pruebas para obtener los parámetros geotécnicos del suelo: tendencias, alcances y limitaciones. Basándose en los ensayos in situ, estos son las más reconocidos y usados en la ingeniería geotécnica: Ensayo SPT, CPT, DPSH, DPL y corte con veleta.

\section{Ensayo in situ}

En la década de los 70 , se presentaron problemas de cimentación en las obras civiles ya sea a poco o largo tiempo de haberse edificado. Entonces para dar solución a ello se hace una exploración geotécnica mediante los ensayos in situ ya que estos van de la mano con los de laboratorio (Díaz-Curiel et al., 2017).

\section{Ensayo SPT}

El ensayo de penetración SPT es uno de los más utilizados en todas partes del mundo para investigaciones de subsuelos (DeJong et al., 2017). Este ensayo, denominado en inglés Standard Penetration Test dio inicio a la exploración de suelos en el año 1927 en los Estados Unidos cuando el sondista Raymond Concrete Pile hizo la propuesta a Terzaghi para realizar el conteo de la cantidad de golpes en el momento de hincar un pie de la toma muestra en terrenos no cohesivos (Abou-matar y Goble, 1997).

Por largo tiempo, se han realizado una gran cantidad de ensayos Terzaghi y Peck. En el año 1948, todos los resultados obtenidos mediante el equipo de penetración fueron publicados en uno de sus libros denominado "Mecánica de suelos en la ingeniería practica" ( Robertson, Campanella, y Wightman, 1983).

Según Sánchez-Ávila et al. (2018) por naturaleza, el ensayo es muy simple y puede ser aplicado en cualquier tipo de terreno con mucha facilidad. Para hacer una exploración geológica con este ensayo, se ubica la broca hasta la altura esperada y dentro de ella en la base se coloca la toma muestra que puede ser de dimensiones variadas y está compuesta por tres elementos: primero, la zapata, luego tenemos al tubo bipartido y por último la cabeza de acoplamiento en el varillaje (Changho et al., 2010).

Se llega a hincar la toma muestra sobre el terreno $60 \mathrm{~cm}$ contando la cantidad de golpes que sea obligatorio para hincar en un intervalo de $15 \mathrm{~cm}$. Esta hincada se realiza mediante un equipo sólido pesado 
de 63.5 kilogramos que cae desde una altura de $76.2 \mathrm{~cm}$ ante una parte plana llamada yunque, correspondiendo la caída en un trabajo que es teórico de $0.5 \mathrm{KJ}$ por golpe (Abou-matar y Goble, 2004). Por otra parte, se especifica que en este tipo de ensayos se recomienda la corrección del valor a la resistencia a penetración estándar siendo esta con una profundidad más de 20m (Daniel et al., 2006).

Odebrecht et al. (2005) especifica que este ensayo es, especialmente, para los terrenos que contengan una amplia fracción arenosa y por otra parte esta prueba es menor cuando hay existencia de una gran proporción de finos o de gravas. De tal forma que el SPT está difundido por todo el mundo y existen variedades de estudios que permiten esta relación con ecuaciones empíricas del $\mathrm{N}_{30 \text { SPT }}$ ante las muestras que se han obtenido del terreno in situ (Dean y Mohammed, 2014).

La mayoría de las correlaciones pertenecen exactamente a la exploración geológica de terrenos arenosos por lo que la apariencia de grava perturba los resultados o también esta puede impedir la realización del ensayo (Ghafghazi et al., 2014).

Respecto a las correlaciones en el número de golpes de $\mathrm{N}_{\mathrm{SPT}}$, Terzaghi $\mathrm{y}$ Peck en 1948 fueron los primeros en la publicación de correlación con la densidad relativa de las arenas cuarcíticas donde las muestras han sido obtenidas en terrenos in situ (SCHNAID et al., 2007).

En 1986, Skemtom propone factores de corrección en la que estos valores están en función de la profundidad de prueba y del diámetro de pozo, pero dichas ratificaciones son empleadas para los suelos granulares, por lo que es despreciable para los suelos cohesivos (Ghafghaz et al., 2017).

\section{Ensayo de CPT}

El ensayo de penetración CPT: la evaluación geológica, con este método, se obtiene utilizando las muestras de manera rápida y precisa para determinar las propiedades del suelo (Tomás et al., 2007). En 1932, en Holanda fue donde se dio inicio al trabajo con el primer cono para determinar las propiedades del suelo. La labor fue ejecutada por P. Berentsen del departamento de Obras Públicas (Fernández-Diéguez et al., 2016).

T. K Huizinga fue el primer Director de Mecánica de Suelos de Delft-Holanda. Él fue quien construyó 10 toneladas para empujar el primer cono. En Europa desde 1950 emplearon mucho la prueba CPT donde obtuvieron buenos resultados en estudios de los suelos finos (Mora, 2013). Y en el año 1975 Tortensson de Suecia y Wissa de Estados Unidos realizaron los piezómetros electrónicos que permiten medir las presiones de poros durante la penetración y durante las pausas en la penetración (Fernández-Naranjo et al., 2014).

Después en el año 1981, en San Luis Missouri, se realizó una sesión sobre el ensayo CPT que fue organizado por ASCE. Muchos investigadores presentaron sus resultados de dicho ensayo siendo esta medida por la resistencia de punta, la fricción y las presiones de poros de una forma simultánea (Vega-Posada et al., 2017).

El ensayo denominado en inglés Cone Penetration Test. Este se realiza 
mediante el hinca a presión sobre el suelo con una velocidad constante. También mide el esfuerzo que es necesario para la penetración del cono. Esta prueba, por lo general, se realiza en suelos finos. No es recomendable para suelos con grava, suelos cementados y rocas, estos pueden malograr el equipo y el ensayo es rechazado (Ballesteros, 2018).

El ensayo está compuesto por una punta cónica con sección de 10 y $15 \mathrm{~cm}^{2}$ en un ángulo de apertura de $60^{\circ}$ y con una amplificación cilíndrica exterior de 35.7 $\mathrm{mm}$ de diámetro. En la parte superior de la punta cónica se sitúa el friction sleeve. $\mathrm{Su}$ función en mensurar el rozamiento lateral específico (Pastor et al., 2018).

\section{Ensayo DPSH}

El ensayo de penetración dinámica DPSH (Dynamic Probing Super Heavy) es utilizado por la energía que proporciona. Es más alto y puede reconocer una mayor profundidad de terrenos resistentes. Es un tipo de sondeo dinámico que se usa ampliamente para determinar la resistencia al soporte del suelo, suelos arenosos o arcillosos (Wang et al., 2016)

También se utilizan perforaciones para la instalación de instrumentación de monitoreo. La prueba DPSH (súper peso) dividida en masa A de $63,5 \mathrm{~kg}$, altura de caída $50 \mathrm{~cm}$ (muy similar a la antigua Borros, Suecia) y B, con un peso de $63,5 \mathrm{~kg}$. La altura descendente es de $75 \mathrm{~cm}$, en realidad equivalente al SPT, pero el conjunto del dispositivo de golpeo no puede superar $115 \mathrm{~kg}$. El punto o cono de penetración muestra un ángulo de embate de $90^{\circ}$ y está incorporado solamente al varillaje. La puntaza de penetración consigue ser rescatada o disipada (Grana y Tommasi, 2014).

El recuento de golpes DPSH se cuenta por encima de $300 \mathrm{~mm}$ y se denomina valor $\mathrm{N}_{305 \mathrm{~B}}$. Se calcula el número de golpes necesarios para empujar con la puntaza en el terreno de $20 \mathrm{~cm}$ hacia el suelo, lo que se llama N20. Cuando tres valores consecutivos son iguales o superiores a 75 golpes, un valor llega a los 100 golpes o más, o la fricción de la varilla es superior a 200 N.M, la prueba finaliza (MacRobert, 2017).

La acción de cementación entre las partículas del suelo está determinada, principalmente, por la temperatura del suelo por lo que los conteos de golpes de DPSH en el suelo de relleno aumentan rápidamente y la fuerza de cementación correspondiente es alta cuando el suelo se congela, pero los conteos de golpes disminuyen drásticamente. y la fuerza de cementación correspondiente es pequeña una vez que el suelo se descongela (Wang et al., 2016). Este ensayo se rige mediante la norma UNE 103-801.

\section{Ensayo DPL}

El ensayo de penetración ligero consiste en determinar la resistencia del suelo, siendo un equipo liviano y de fácil uso que permite ver la capacidad de soporte in situ ante la realización de una estructura o de la ejecución de una obra civil (Žaržojus y Dundulis, 2010).

Scale en el año 1956 realizó este ensayo denominado DPL en el cual los investigadores LIvneh y Ishali de 1987 y Keleyn de 1975 efectuaron estudios básicos en campo para la evaluación de pavimentos (Ampadu y Dzitse-Awuku, 
2009). Se especifica que el Ensayo de penetración Dinámica Ligero es utilizado en lugares como Inglaterra, Australia, Canadá, Nueva Zelanda y Estados Unidos (Aral y Gunes, 2017).

El DPL está formado por una punta de cónica metálica de $60^{\circ}$, y para la aplicación de los golpes cuenta con un martillo de $10 \mathrm{~kg}$ donde esta se deja caer desde una altura de $50 \mathrm{~cm}$ (Ahmed, Agaiby y AbdelRahman, 2013). La prueba del DPL es un equipo que se puede transportar con facilidad y evalúa la capacidad de resistencia de explanadas y las capas granulométricas de caminos, carreteras y pistas de aeropuertos (Khodaparast et al., 2019).

Esta prueba se denomina en inglés Dynamic Probing Light. Permite obtener muestras para luego evaluar la capacidad portante del subsuelo de una manera directa. También se aplica para rocas (Tarawneh, 2016). De tal forma que la información obtenida es de manera continua, donde el proceso de las mediciones de la resistencia a la penetración se llega a realizar durante todo el proceso de hinca (Akca, 2003).

Para realizar este ensayo se coloca un espécimen de suelo que está sometido por una carga normal (Mehtab et al., 2018). Esta prueba DPL indica acontecimientos de una falla sobre un plano de localización predeterminado en la dirección horizontal, donde actúan dos fuerzas (Elton, 1987).

Con el objetivo de realizar correctamente este ensayo, se debe de verificar si se cuenta con un suelo cohesivo y con respecto a los cálculos se realizan a las $24 \mathrm{~h}$ de haber saturado la muestra (Luján y Aguilar, 2018).

\section{Ensayo corte de veleta}

La incorporación del ensayo de corte de veleta como prueba de campo para estimar la resistencia sin drenaje del suelo fino se remonta a las décadas de 1940 a 1950. Sin embargo, observaron que la resistencia de las arcillas blandas medidas usando VST es significativamente mayor que la intensidad de campo y propusieron factores de corrección empíricos para la resistencia al corte de las paletas para tener en cuenta el efecto de la anisotropía y el efecto de la velocidad del suelo en el campo (Gupta et al., 2016).

La ventaja de esta prueba es que nos permite determinar una resistencia de suelos cohesivos no drenados. El equipo y la prueba son muy sencillos; además, nos permite medir la sensibilidad in situ y se tiene mucha experiencia $y$ conocimiento sobre el uso ( $\mathrm{Ng}$, Yuen, y Dong, 2016).

También Cabalar nos menciona que los resultados de las pruebas de corte de veleta demostraron que los valores de $S_{u}$ obtenidos de las pruebas eran siempre más altos que los alcanzados de las pruebas de cono de caída (Cabalar, Mukhlif, y Isik, 2020).

\section{Estado actual}

La prueba de Penetración SPT es uno de los ensayos más usados y extendidos, actualmente, en la geotecnia por su sencillez, rapidez y bajo costo ( Sil y Haloi, 2017). Pero se llega a valorar que un $85 \%$ a $90 \%$ de los diseńos empleados en las cimentaciones convencionales del Norte y Sur América. Se basa en la medición del valor $\mathrm{N}$ de la prueba SPT. (Thokchom et al, 2017) 
En cambio, el Ensayo de Penetración de Cono (CPT) es una de las técnicas de caracterización más utilizadas de suelos sensitivos, pero a diferencia del ensayo SPT el CPT brinda intervalos más cortos e información más confiable, donde posibilita instituir perfiles exactos y demostrar pequeños cambios que estas pueden suceder entre las capas de suelos, y también posee un costo elevado (MolaAbasi et al., 2017).

Por otra parte, la prueba de penetración DPL es un ensayo de penetración dinámica ligero de operación manual que permite conocer profanidades menores a $10 \mathrm{~m}$, siendo de uso muy fácil y manejable para determinar la resistencia del suelo y rocas. (Santos y Bicalho, 2017).
Pero la prueba de ensayo de corte con veleta solo se realiza en suelo cohesivo no perturbado y remoldado. El método de corte de veleta es adecuado para suelos arcillosos saturados porque los resultados serán diferentes si se realiza en otros suelos. (Olguín y Ortúzar, 2015)

No hay duda de que las pruebas de penetración dinámica súper pesada DPSH son las que más se utilizan, probablemente, porque proporcionan mayor energía y pueden identificar mayores profundidades y terrenos más resistentes (Khodaparast, Rajabi y Mohammadi, 2015).

Tabla 1

\section{Comparación de las ventajas y limitaciones entre los distintos tipos de ensayos in situ}

\begin{tabular}{|c|c|c|c|c|c|}
\hline & SPT & $\mathrm{CPT}$ & DPSH & DPL & Veleta \\
\hline Tipos de suelos & La mayoría & Gravas no & $\begin{array}{c}\mathrm{La} \\
\text { mayoría }\end{array}$ & La mayoría & $\begin{array}{c}\text { Cohesivos } \\
\text { no } \\
\text { drenados } \\
\end{array}$ \\
\hline Continuidad /perfil & No & $\begin{array}{c}\text { Sí, datos } \\
\text { cada } 10 \text { o } 20 \\
\mathrm{~cm} \\
\end{array}$ & $\begin{array}{l}\text { Sí, datos } \\
\text { cada } 20 \\
\mathrm{~cm}\end{array}$ & No & No \\
\hline Toma de muestra & Sí & No & No & Sí & No \\
\hline Repetitividad & Buena & Muy buena & Buena & Buena & Regular \\
\hline $\begin{array}{l}\text { Sensibilidad a cambios } \\
\text { en el perfil estratigráfico }\end{array}$ & Regular/Buena & $\begin{array}{c}\text { Buena/Muy } \\
\text { buena }\end{array}$ & Buena & Regular/buena & Regular \\
\hline $\begin{array}{c}\text { Correlaciones empíricas } \\
\text { para determinar } \\
\text { propiedades del suelo }\end{array}$ & Sí & Sí & Sí & - & - \\
\hline $\begin{array}{l}\text { Interpretación teórica } \\
\text { para determinar } \\
\text { propiedades del suelo } \\
\end{array}$ & No & Sí & No & - & - \\
\hline $\begin{array}{l}\text { Posibilidad de otros } \\
\text { captores o sensores }\end{array}$ & No & No & No & - & - \\
\hline
\end{tabular}

Nota. (Devincenzi y Frank, 2004)

En la Tabla 1, se muestra una comparación de los ensayos in situ mencionados, especificando el tipo de suelo donde es aplicado, la continuidad al momento de tomar la muestra, la repetividad, la sensibilidad a cambios en el perfil estratigráfico. 


\section{Conclusiones}

En el mundo dela ingeniería geotécnica hay varias maneras para determinar las características de los suelos siendo estas desde la exploración del suelo en la obtención de muestras mediante las pruebas in situ para luego llevarse a un laboratorio y ser evaluadas.

Los ensayos in situ son muy importantes en la obtención del suelo, y con respecto a su utilización deben ser aplicados por operadores especializados en dichos equipos para evitar daños durante la ejecución, como también impedir malograr el equipo, y de esta manera obtener los resultados que sea de gran éxito.

Es de importancia la exploración del suelo ya que de esta manera permite evaluar el terreno y ejecutar una obra civil dependiendo del tipo de diseño que se va a realizar.

\section{Referencias}

Abou-matar, H., \& Goble, G. (1997). SPT Dynamic analysis and measurements. Journal of Geotechnical and Geoenvironmental engineering, $921-928$. h t t p s:// d o i. org/10.1061/(ASCE) 1090 0241(1997)123:10(921)

Abou-matar, H., \& Goble, G. (2004). SPT Dynamic Analysis and Measurements. American Society of Civil Engineers, 441-462. https://doi. org/10.1061/40743(142)25

Ahmed, S., Agaiby, S., \& Abdel-Rahman, A. (2013). A unified CPT-SPT correlation for non-crushable and crushable cohesionless soils. Ain Shams Engineering Journal, 1-11. https://doi.org/10.1016/j. asej.2013.09.009

Akca, N. (2003). Correlation of SPT-CPT data from the United Arab Emirates. Engineering Geology, 219-231. https://doi.org/10.1016/S00137952(02)00181-3
Ampadu, S., \& Dzitse-Awuku , D. (2009). Model tests for bearing capacity in a lateritic soil and implications for the use of the dynamic cone penetrometer. International Conference on Soil Mechanics and Geotechnical Engineering, 1076-1079. http://ebooks. iospress.nl/publication/30146

Aral , F., \& Gunes, E. (2017). Correlation of Standard and Cone Penetration Tests: Case Study from Tekirdag (Turkey). IOP Conference Series: Materials Science and Engineering, 1-9. https:// iopscience.iop.org/article/10.108 8/1757-899X/245/3/032028

Ballesteros, R. (2018). Obtención de parámetros del modelo Hardening soil mediante ensayo CPTu en suelos blandos de Bogotá. Revista Politécnica, 89-97. https://revistas.elpoli.edu.co/index.php/pol/ article/view/999

Cabalar, A., Mukhlif, M., \& Isik, H. (2020). A comparative study on 
the undrained shear strength results of fall cone and vane shear tests in sand-clay mixtures. Arabian Journal of Geosciences, 1-11. https://doi.org/10.1007/s12517020-05351-5

Changho, L., Jong-Sub, L., Shinwhan, A., \& Woojin, L. (2010). Effect of Secondary Impacts on SPT Rod Energy and Sampler Penetration. Journal of Geotechnical and Geoenvironmental Engineering, 522526. https://doi.org/10.1061/ (ASCE)GT.1943-5606.0000236

Çoşkun, G., Sarışık, G., \& Sarışık, A. (2017). Slip Safety Risk Analysis of Surface Properties Using the Coefficients of Friction of Rocks. International Journal of Occupational Safety and Ergonomics, 1-25. https://doi.org/10.1080/10 803548.2017.1395594

Daniel, C., Howie, J., Scott, R., \& Walker, B. (2006). Discussion of "Review of Standard Penetration Test Short Rod Corrections" by Chris R. Daniel, John A. Howie, R. Scott Jackson, and Brian Walker. Journal of Geotechnical and Geoenvironmental Engineering, 1637-1640. https:// doi.org/10.1061/(ASCE)10900241(2006)132:12(1637)

Dean, E., \& Mohammed, R. (2014). Simplified Shockwave Analysis of the Standard Penetration Test. International Journal of Geomechanics, 1-13. https://doi. org/10.1061/(ASCE)GM.19435622.0000379
DeJong, J., Ghafghazi, M., Sturm, A., Wilson, D., Dulk, J., Armstrong, R., . . . Davis, C. (2017). Instrumented Becker Penetration Test. I:Equipment, Operation, and Performance. Journal of Geotechnical and Geoenvironmental Engineering, 1-12. https://doi. org/10.1061/(ASCE)GT.19435606.0001717

Devincenzi, M., \& Frank, N. (2004). Ensayos Geotecnicos In situ su ejecucion e interpretacion. IGEOTEST,S.L. http://www.estudiosgeotecnicos.info/wp-content/ uploads/2014/06/Devicenci-Ensayos-in-situ.pdf

Díaz-Curiel, J., Rueda-Quintero, S., Biosca, B., \& Doñate-Matilla, G. (2017). Advance in the penetrometer test formulation to estimate allowable pressure in granular soils. Acta Geotechnica, 1-9. https://link.springer.com/article/10.1007\%2Fs 1 1440-0 17-0565-x\#article-info

Díaz-Curiel, J., Rueda-Quintero, S., Biosca, B., \& Doñate-Matilla, G. (2017). Advance in the penetrometer test formulation to estimate allowable pressure in granular soils. Acta Geotechnica, 1-9. https://doi.org/10.1007/s11440017-0565-x

Elton, D. (1987). Settlement of Footings on Sand by CPT Data. American Society of Civil Engineers, 99113. https://doi.org/10.1061/ (ASCE)0887-3801(1987)1:2(99) 
Fernández-Diéguez, L., GuardadoLacaba, R., Herrera-Delfín, I., Oliva-Álvarez, R., \& Díaz-Santo , P. (2016). Escenarios susceptibles a la licuefacción inducida por sismos de gran magnitud en Santiago de Cuba. Minería y Geología, 53-69. https://www.redalyc.org/articulo. oa?id=223545820004

Fernández-Naranjo, F., Rodríguez, V., Rodríguez, R., Alberruche, M., Arranz , J., \& Vadillo, L. (2014). Liquefaction susceptibility assessment and study of "La Luciana" tailings dam fault (Spain, 1960) based on historical documents. DYNA, 189-198. https://dialnet. unirioja.es/servlet/articulo?codi$\mathrm{go}=6191295$

Ghafghaz, M., DeJong, J., Sturm, A., \& Temple, C. (2017). Instrumented Becker Penetration Test. II: iBPTSPT Correlation for Characterization and Liquefaction Assessment of Gravelly Soils. American Society of Civil Engineers, 1-10. https://doi.org/10.1061/(ASCE) GT.1943-5606.0001718

Ghafghazi, M., Thurairajah, A., DeJong, J., Wilson, D., \& Armstrong, R. (2014). Instrumented Becker Penetration Test for Improved Characterization of Gravelly Deposits. American Society of Civil Engineers, 37-46. https://doi. org/10.1061/9780784413272.004

Grana, V., \& Tommasi, P. A. (2014). A deep-seated slow movement controlled by structural setting in marly formations of Central Italy. Landslides, 11, 195-212. doi:h- ttps://doi.org/10.1007/s10346013-0384-6

Gupta, T., Chakraborty, T., K., A.-R., \& Achmus, M. (2016). Large Deformation Finite Element Analysis of Vane Shear. Geotechnical and Geological Engineering, 34, 1669-1676. doi:https://doi. org/10.1007/s10706-016-0048-0

Hipólito-Ojalvo, F., Zamora-Polo, F., Luque, A., \& Naharro-Saqueda, I. (2019). Evaluación de las propiedades del subsuelo de Badajoz (España) con fines constructivos mediante Sistemas de Información Geográfica. Informes de la Construcción, 71, 1-9. http://informesdelaconstruccion.revistas. csic.es/index.php/informesdelaconstruccion/article/view/5973

Khodaparast, M., Rajabi, A., \& Derakhshan, M. (2019). Development of Practical Correlations Between Cone Penetration Resistance and SPT Values for Various Types of Soils. Iranian Journal of Science and Technology, Transactions of Civil Engineering, 1-11. https://doi. org/10.1007/s40996-019-00319-2

Khodaparast, M., Rajabi, A., \& Mohammadi, M. (2015). The new empirical formula based on dynamic probing test results in fine cohesive soils. International Journal of Civil Engineering, 105-113. http://ijce.iust.ac.ir/article-1 1309-en.html

Lingwanda, M., Larsson, S., \& Nyaoro, D. (2015). Correlations of SPT, CPT and DPL Data for Sandy 
Soil in Tanzania. Geotech Geol Eng, 1221-1233. https://doi. org/10.1007/s10706-015-9897-1

Luján, E., \& Aguilar, R. (2018). Utilización de Métodos Experimentales y de Simulación Numérica para el estudio de la Microzonificación Sísmica del distrito de Trujillo, provincia Trujillo, departamento La Libertad, Perú. Revista CIENCIA Y TECNOLOGÍA, 37-49. https://revistas.unitru.edu.pe/index.php/PGM/article/view/2105

MacRobert, C. (2017). Interpreting DPSH penetration values in sand soils. Journal of the South African, 59(3), 11-15. doi:http://dx.doi. org/10.17159/2309-8775/2017/ v59n3a2

Mehtab, A., Muhammad, A., Shamsher, S., Saeedullah , J., Muhammad, B., Maqsood-ur-Rehman, \& Nawaz, A. (2018). Empirical SPT-CPT correlation for soils from Lahore, Pakistan. IOP Conference Series: Materials Science and Engineering, 1-7. https:// iopscience.iop.org/article/10.108 8/1757-899X/414/1/012015

Mola-Abasi, H., Kordtabar, B., \& Kordnaei, A. (2017). Liquefaction prediction using CPT data by triangular chart identification. International Journal of Geotechnical Engineering, 1-6. https://doi.org/ $10.1080 / 19386362.2017 .12823$ 99

Mora, R. (2013). Uso de métodos estadísticos para la identificación de capas de suelos volcánicos con el ensayo del cono de penetración en los terrenos de la Universidad de Costa Rica, Montes de Oca, San José, Costa Rica. Revista Geológica de América Central, 109-120. https://revistas.ucr.ac.cr/index.php/geologica/article/ view/13107

Odebrecht, E., Schnaid, F., Maia, M., \& Paula, G. (2005). Energy Efficiency for Standard Penetration Tests. Journal of Geotechnical and GeoenvironmentaL Engineering, 1252-1263. https:// doi.org/10.1061/(ASCE) 10900241(2005)131:10(1252)

Olguín , R., \& Ortúzar, M. (2015). Desarrollo e implementación de una veleta de corte a alta revolución para sondajes. Obras y Proyectos, 8995. https://scielo.conicyt.cl/ scielo.php?script=sci_arttext\&pi$\mathrm{d}=$ S0718-28132015000100011\&l$\mathrm{ng}=\mathrm{en} \& \mathrm{nrm}=\mathrm{iso} \& \mathrm{t} \operatorname{lng}=\mathrm{en}$

Pastor, J., Tomás, R., Cano, M., \& Riquelme, A. (2018). Estudio comparativo del potencial de licuación de suelos usando las normas españolas y el Eurocódigo. Boletín de la Sociedad Geológica Mexicana, 761-778.

https://www.redalyc.org/articulo. oa? id=94358881009

Reyes-Ortiz, O. (2009). Cambios dinámicos y mecánicos de una mezcla asfáltica densa por las propiedades del asfalto y la energía de compactación. Ingeniería \& Desarrollo, 139-155. http://web.b.ebscohost. $\mathrm{com} /$ ehost $/$ detail/detail? vid=2\&sid $=8$ be 5 f669-fed $2-41$ ba- 8 f03- 
358239 bd e $597 \% 40$ p $\mathrm{dc}$-v-sessmgr02\&bdata $=$ Jmxhbmc9ZXMmc210ZT11aG9zdC1saXZl\#AN=52316454\&db=zbh

Robertson, P., Campanella, R., \& Wightman, A. (1983). SPT-CPT Correlations. Journal of Geotechnical Engineering, 1449-1459. https:// doi.org/10.1061/(ASCE)07339410(1983)109:11(1449)

Sánchez-Ávila, D., Barea, R., Martínez, E., Ramón, J., Portolés, L., \& Carreño, F. (2018). Determination of the instantaneous strain rate during small punch testing of 316L stainless steel. International Journal of Mechanical Sciences, 3-25. https://doi.org/10.1016/j. ijmecsci.2018.09.042

Santos, M., \& Bicalho, K. (2017). Proposals of SPT-CPT and DPLCPT correlations for sandy soils in Brazil. Journal of Rock Mechanics and Geotechnical Engineering, 1-7. https://doi.org/10.1016/j. jrmge.2017.08.001

Schnaid, F., Odebrecht, E., \& Rocha, M. (2007). On the mechanics of dynamic penetration tests. Geomechanics and Geoengineering: An International Journal, 137-146. https://doi.org/10.1080/17486020 701383825

Skempton, A. (1986). Standard penetration test procedures and the effects in sands of overburden pressure, relative density, particle size, ageing and overconsolidation. Géotechnique, 425-447. https://doi. org/10.1680/geot.1986.36.3.425
Sil, A., \& Haloi, J. (2017). Empirical Correlations with Standard Penetration Test (SPT)-N for Estimating Shear Wave Velocity Applicable to Any Region. Int. J. of Geosynth. and Ground Eng., 1-13. https:// doi.org/10.1007/s40891-0170099-1

Tarawneh, B. (2016). Predicting standard penetration test $\mathrm{N}$-value from cone penetration test data using artificial neural networks. Geoscience Frontiers, 1-6. https://doi. org/10.1016/j.gsf.2016.02.003

Thokchom, S., Rastogi, B., Dogra, N., Pancholi, V., Sairam, B., Bhattacharya, F., \& Patel, V. (2017). Empirical correlation of SPT blow counts versus shear wave velocity for different types of soils in Dholera, Western India. Nat Hazards, 1-16. https://doi.org/10.1007/ s11069-017-2744-3

Tomás, R., Domenech, C., Mira, A., Cuenca, A., \& Delgado, J. (2007). Preconsolidation stress in the Vega Baja and Media areas of the River Segura (SE Spain): Causes and relationship with piezometric level changes. Engineering Geology, 135-151. https://doi.org/10.1016/j.enggeo.2007.01.006

Vega-Posada, C., Ramos-Cañón, A., \& García, E. (2017). Efecto del gas en la velocidad de onda de corte de suelos arenosos densificados con explosivos. Tecnura, 67-80. https://revistas.udistrital.edu. co/index.php/Tecnura/article/ view/11969 
Wang, J., Jia, K., Rafique, R., Guo, L., Yu, Q., Yue, Y., \& Yuan, C. (2016). Changes of backfill soil of tower foundation in the permafrost regions with warm ice-rich frozen soil on the Qinghai-Tibet Plateau. Environmental Earth Sciences, 75, 1-10. doi:https://doi. org/10.1007/s12665-016-6223-z

Žaržojus, G., \& Dundulis, K. (2010). Problems of correlation between dynamic probing test (DPSH) and cone penetration test (CPT) for cohesive soils of Lithuania.
The Baltic journal of road and bridge engineering, 69-75. https:// bjrbe-journals.rtu.lv/article/view/ bjrbe. 2010.10

Žaržojus, G., Kelevišius, K., \& Amšiejus, J. (2013). Energy Transfer Measuring in Dynamic Probing Test in Layered Geological Strata. Procedia Engineering, 57, 1302-1308. https://www.sciencedirect.com/science/article/pii/ S1877705813008977?via\%3Dihub 